\title{
Study on the Detection Method of Electromagnetic Wave Signal under Clutter Jamming
}

\author{
Li Siwei \\ Beijing Institute of Technology 100081
}

Keywords: detection; morphological filtering; distance echo;

\begin{abstract}
During the study process of electromagnetic wave signal detection method under clutter jamming, the influence of noise and interference are not considered when the current algorithm is used to detect electromagnetic wave signal, thus there is problem of large detection error. An electromagnetic wave signal detection method under clutter jamming based on the improved morphological processing algorithm is proposed. The algorithm first combines mathematical morphology filtering and threshold detection, and detects the characteristic parameters, like electromagnetic wave signal electric field strength, magnetic field intensity, polarization flatness, polarization rotation and vector propagation direction, on the basis of it, distance unit echo of larger amplitude in the detection window is regarded as the target echo to judge, and accurately complete electromagnetic wave signal detection under clutter jamming. The experimental results show that the detection method of electromagnetic wave signal based on the improved morphological processing algorithm has the high accuracy and the noise immunity.
\end{abstract}

\section{Introduction}

The electromagnetic radiation phenomenon in ionosphere is very complex, not only related to fluctuations of the earth's magnetic field, but also related to magnetic storm and particle storm of earth space [1.2.3]. Thus, it will produce a lot of electromagnetic disturbance factors, including electromagnetic ion cyclotron waves, whistler waves, plasma layer hiss etc. These clutters influence the transmission of the electromagnetic wave signal seriously, which is easy to cause the failure of electromagnetic wave signal transmission [4.5.6]. Therefore, the detection method of the electromagnetic wave signal under the clutter jamming has received a wide attention, and a lot of good methods are also appeared at the same time.

At present, the mainstream of electromagnetic wave signal detection methods including electromagnetic wave signal detection method under clutter based on wavelet algorithm, electromagnetic wave signal detection method under clutter based on Bayesian algorithm and electromagnetic wave signal detection method under clutter based on neural network algorithm. Among them, the detection method of electromagnetic wave signal based on wavelet algorithm is often used. However, the influence of noise and interference are not considered when these algorithms are used to detect electromagnetic wave signal, thus there is problem of large detection error.

In view of the above problems, an electromagnetic wave signal detection method under clutter jamming based on the improved morphological processing algorithm is proposed. The algorithm has good detection accuracy and strong noise immunity.

\section{Detection principle of electromagnetic wave signal under clutter jamming}

In the detection process of the electromagnetic wave signal under the clutter jamming, it is necessary to detect the characteristic signal which is unique to electromagnetic wave and carry out statistical analysis. Due to the manifestations of different electromagnetic radiation signal in the regional characteristics have some differences, so for ionospheric electromagnetic radiation enhancement usually has the characteristics of certain band width and duration, periodicity of signal and randomness of noise are collected by the receiver, through the operation of auto correlation, the electromagnetic wave signal detection under clutter jamming is completed. The concrete steps are 
as follows:

In the electromagnetic wave signal detection process under cutter jamming, assuming the original containing noise time series obtained by a receiver after removing the mean is $\left\{X_{i}\right\}$, then, periodic signal and random noise are additive, which can be expressed as:

$$
x(n)=s(n)+n(n)
$$

Among them, $s(n)$ is the periodic pulse electromagnetic wave signal, $n(n)$ is the random noise, both of them have the stationary random process which has the ergodic characteristic of each state, and its autocorrelation sequence is:

$$
R_{x x}(m)=R_{s s}(m)+R_{n n}(m)+R_{S n}(m)+R_{n s}(m)
$$

Wherein, $R_{s s}(m)$ is autocorrelation sequence of electromagnetic wave signal, $R_{n n}(m)$ is autocorrelation sequence of noise, $R_{S n}(m)$ is cross-correlation sequence of the electromagnetic wave signal and noise, $R_{n s}(m)$ is cross-correlation sequence of noise and electromagnetic wave signal. If the electromagnetic wave signal and noise are independent statistically, and can be defined as stationary random electromagnetic wave signal, when $m$ is very big, it need to meet the condition $R_{n n}(m)=\sigma^{2} e^{-\alpha[m]} \rightarrow 0$, and autocorrelation sequence of the electromagnetic wave signal with cycle $T$ is still the sequence with cycle $T$. Therefore, when $m$ is large, $R_{x x}(m)$ is approaching $R_{s s}(m)$.

\section{The correlation principle of the electromagnetic wave signal detection optimization method under the clutter jamming}

\subsection{Optimization detection of threshold}

In optimization detection process of electromagnetic wave signal under clutter jamming, The algorithm first combines mathematical morphology filtering and threshold detection, and detects the characteristic parameters, like electromagnetic wave signal electric field strength, magnetic field intensity, polarization flatness, polarization rotation and vector propagation direction. The concrete steps are as follows:

In the process of the optimization detection of electromagnetic wave signal under clutter jamming, the threshold selection can be determined according to the empirical values, and the threshold of the magnetic field is determined as:

$$
T h B=-75 \lg (p T)^{2} / H_{Z}
$$

In the process of the optimization detection of electromagnetic wave signal under clutter jamming, the threshold of the electric field power spectrum can be obtained according to the formula:

$$
T h E=0.4 \lg (\mu V / m)^{2} / H_{Z}
$$

In the process of the optimization detection of electromagnetic wave signal under clutter jamming, the above equation is utilized to detect the electromagnetic radiation of the planar polarization degree over 0.7 , which is $T h F e \geq 0.7$.

In the process of the optimization detection of electromagnetic wave signal under clutter jamming, the basic operation of morphology is divided into expansion, corrosion, open operation and close operation. A large number of redundant information is removed by threshold segmentation, then the signal parameters of electromagnetic wave signal can be described as two valued signals, assuming that the set $A$ and $B$ are contained in space $Z^{2}$, and the definition of $A$ is expanded by $B$ is :

$$
A \oplus B=\left\{z \mid(\tilde{B})_{z} \cap A \neq \phi\right\}
$$

In the formula, $A$ is the input signal, and $B$ is the structural element. $A$ is expanded by $B$ 
means that $\tilde{B}_{z}$ is obtained the $B$ after reflection shift $z$ relative to its origin..

Similarly, the corrosion operation is expressed as $A B$, defined as:

$$
A \Theta B=\left\{z \mid(B)_{z} \subseteq A\right\}
$$

In the process of the optimization detection of electromagnetic wave signal under clutter jamming, the open operation and closing operation are expressed as:

$$
\begin{aligned}
& A \cdot B=(A \oplus B) \Theta B \\
& A \circ B=(A \Theta B) \oplus B
\end{aligned}
$$

3.2 The realization of the optimization method of electromagnetic wave signal detection under the clutter jamming

In the process of the optimization detection of electromagnetic wave signal under clutter jamming, assuming $\alpha_{k}\left(k \in \Theta_{j}\right)$ represents target scattering center electromagnetic wave echo amplitude, when extended target is detected in the echo data, $\alpha_{k}\left(k \in \Theta_{j}\right)$ need to be estimated. Assuming that $\alpha_{k}\left(k \in \Theta_{j}\right)$ is kept constant in every pulse, the following equation can be obtained when the covariance matrix of the clutter is known:

$$
\lambda_{g I R T}=\frac{\max _{\alpha k} \prod_{k \in \Theta j} h_{c}\left(q_{1}(k)\right)}{\prod_{k \in \Theta j} h_{c}\left(q_{0}(k)\right)} \ll G_{g I R T}
$$

Among them, $G_{g l R T}$ is the detection threshold.

Using the distance unit of larger amplitude in electromagnetic echo to detect extended target, statistics $\omega_{k}$ is arranged in descending order to get the equation:

$$
\omega_{(1)} \geq \omega_{(2)} \geq \ldots \geq \omega_{(k)} \geq \omega_{(K)}
$$

In the process of the optimization detection of electromagnetic wave signal under clutter jamming, the former $J$ statistics are utilized to meet the condition $\omega_{(K)}(k=1,2, \cdots, J)$, the electromagnetic wave signal detection equation is obtained.:

$$
\lambda_{\text {OS-GLRT }}=-2(N-1) \sum_{K=1}^{J} 1 n\left(1-\omega_{(k)}\right) \succ_{\prec} G_{0 S-G L R T}
$$

In the above formula, the detection threshold in the electromagnetic wave signal optimization detection process under clutter jamming is represented by $G_{0 S-G L R T}$.

\section{Experiments and simulation results}

In order to prove the validity of the detection method of the electromagnetic wave signal under the clutter jamming based on the improved morphological processing algorithm, an experiment is needed. The Monte Carlo simulation method is adopted for electromagnetic wave signal detection under clutter jamming based on wavelet algorithm, the improved algorithm, Bayesian algorithm, detection time and accuracy of different algorithms are compared to measure electromagnetic wave signal detection performance of three algorithms.

Experiment 1)

The time needed for 9999 times Monte-Carlo simulation experiments of three detection algorithms are listed in Table 1:

Table 1 Detection time of different algorithms

Algorithm Wavelet algorithm $\quad$ Improved algorithm $\quad$ Bayesian algorithm

$\begin{array}{llll}\text { Detection time/S } & 35.45 & 39.23 & 12.19\end{array}$




\section{Experiment 2)}

The detection accuracy in the Monte-Carlo simulation experiment of three different algorithms are compared, and the results are shown in the following image:

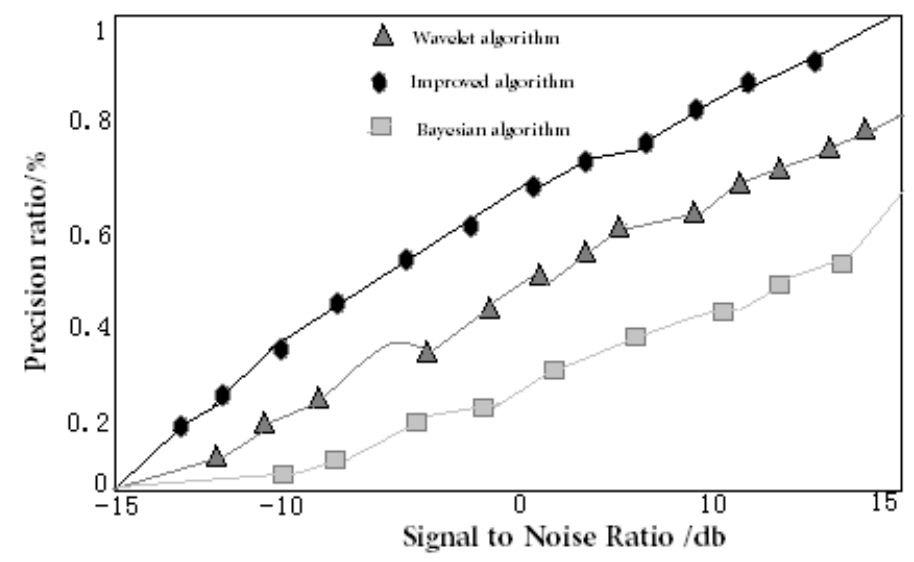

Fig. 1 Comparison of the detection accuracy of different algorithms

It can be seen from table 1 and figure 1, when the optimized algorithm is used for detection accuracy in Monte Carlo simulation experiments, the running time of the optimized algorithm is superior to the other two algorithms, since the algorithm combines mathematical morphology filtering and threshold detection, and detects the characteristic parameters, like electromagnetic wave signal electric field strength, magnetic field intensity, polarization flatness, polarization rotation and vector propagation direction, on the basis of it, distance unit echo of larger amplitude in the detection window is regarded as the target echo to judge, and accurately complete electromagnetic wave signal detection under clutter jamming.

\section{Conclusions}

In view of the influence of noise and interference are not considered when the current algorithm is used to detect electromagnetic wave signal, there is problem of large detection error. An electromagnetic wave signal detection method under clutter jamming based on the improved morphological processing algorithm is proposed. The algorithm first combines mathematical morphology filtering and threshold detection, and detects the characteristic parameters, like electromagnetic wave signal electric field strength, magnetic field intensity, polarization flatness, polarization rotation and vector propagation direction, on the basis of it, distance unit echo of larger amplitude in the detection window is regarded as the target echo to judge, and accurately complete electromagnetic wave signal detection under clutter jamming. The experimental results show that the detection method of electromagnetic wave signal based on the improved morphological processing algorithm has the high accuracy and the noise immunity.

\section{References:}

[1] Li Cuiyun, Jiang Zhou, Ji Hongbing. A new P HD filters under unknown complex wave [J].Journal of Xi'an University of Electronic Science and technology.2014.5:18-23.

[2] Xiu Jianjuan, Wang Wangsong, He You. Multiple target tracking in clutter based on distance speed and course [J]. Systems engineering and electronics.2014.9:1702-1706.

[3] Zhang Dandan, Qiu Xiaolan, Hu Donghui, Ding Chibiao. Analysis of Geosynchronous Satellite-Air Bistatic SAR Clutter Characteristics from the Viewpoint of Ground Moving Target Indication [J]. Journal of Radars.2013.3:348-356.

[4] Zhang Xin, Cui Chen, Yu Jian. Multiple extended targets tracking for cognitive radar in the presence of signal-dependent clutter [J]. Advances in New and Renewable Enengy, 2013.2:492-499.

[5] Zhao Yinan, Li Fengcong, Yin Bin. Adaptive Polarimetric Detection of Targets in Heavy-tailed 
Compound-Gaussian Clutter [J]. Electronics and information of.2013.2:376-380.

[6] Song Jie, Liu Ningbo, Wang Guoqing, Guan Jian, He You. Approximate Fractality of Sea Clutter FRFT Spectrum and Target Detection [J]. Journal of Astronautics, 2013.10:1394-1402.

[7] Yang Jinghui, Gao Jinbao, Zhang Xiaolin. Research on Noisy Mixed Communication Signals Separation and Identification [J]. TV technology.2013.11:173-176.

[8] Zhao Jie, Hua Gang, Yu Bo. De-noising Method of Mine Wire Rope Damage Signal Based on Improved Wavelet Threshold Algorithm [J]. Coal mine machinery, 2013, 34 (1): 284-286.

[9] Li Qinghua, Senbai Dalabaev, Quan Fusun. Brige-Massart Policy of Application of ECG Based on Wavelet Threshold De-Noising [J]. Computer simulation, 2013, 30 (10): 368-371.

[10] Feng Ganzhong, He Zhiming. Wideband Radar Signal Denoising Method Based on Modulus Maximum [J]. Modern radar, 2013, 35 (6): 38-41. 